\title{
Broad Environmental Change Blindness in Virtual Environments and Video Games
}

\author{
Daryl Marples ${ }^{1[0000-0003-2583-2893]}$, Pelham Carter $^{2[0000-0002-6132-1612], ~}$ \\ Duke Gledhill[10000-0002-7260-831X] and Simon Goodson ${ }^{1[0000-0002-0939-0629]}$ \\ ${ }^{1}$ University of Huddersfield, West Yorkshire, HD1 3DH, UK \\ ${ }^{2}$ Birmingham City University, Birmingham, B4 7BD, UK \\ d.marples@hud.ac.uk
}

\begin{abstract}
For almost 120 years, it has been demonstrated in literature that humans are susceptible to many different types of change blindness; essentially if a change in a visual scene is not sudden or obvious, it fails to be detected. Research utilising video game environments provides the opportunity for the study of change blindness during dynamic and interactive tasks. This study examined participant perception of gradual broad changes to both colour and textural information across surfaces and objects in a video game mimicking that of a FirstPerson adventure or point and click game/genre escape room task. 119 participants were asked to solve a room escape puzzle, results demonstrated that perception of gradual textural changes to an environment across a range of decreasing durations (90s, 45s, 22.5s \& 11.25s) were detected only by a very small proportion of participants. (4.20\%). There was no significant effect of the variation in time on detection rates.
\end{abstract}

Keywords: Attention, Change Blindness, Virtual, Escape Room, Change Detection.

\section{Introduction}

When a magician makes a coin 'disappear' a technique of misdirection is used to infer the transfer of the coin and holding that attention allows for the discreet, [1], [2] similarly film makers take advantage of the phenomenon that minor differences in a scene between cuts are generally not perceived by the viewer. This is despite millions of neurons attending to the scene and ready and waiting to react should they detect change [3].

In the 1980's psychologists became interested in these phenomena [4], [5] and over the intervening decades, a variety of types of change blindness [6] have been identified these include, blinks or eye motion [7], flashed blank images [8], or gradual subtle changes over time [4], [9]. 
It has been demonstrated that the limitations of working memory have direct correlations to the perception of colours relating to objects [10]. It has further been demonstrated that specific non-colour details pertaining to an image are recalled in more depth if that image is presented in monochrome thus reducing the memory load of storing colour data [11]. In relation to this, in attention tests with multiple differing coloured objects, where one is covered, few participants correctly identified the correct colour of the hidden object [12]. The reasons behind this are related to selective awareness governed by two visual input pathways [13], a 'selective' pathway that governs object recognition and is responsible for recognition of one or a very small number of objects at a time and a 'non-selective pathway' which is responsible for forming a broader impression of a scene, this system may give a broad understanding that there are objects in a scene, but not what those objects actually are, nor individual characteristics such as colour.

From a neurological perspective, the fact that these interweaving systems appear to operate in a semi-autonomous manner can be related to the fact that it has been demonstrated that storage and processing of different types of sensory data are handled independently within the structure of the brain, [14], [15]. The specific subcomponent of working memory connected to the retention of visual information is Visual Short-Term Memory (VSTM) [16]. However, for an element of a scene to be recorded in VSTM, it first must be attended to [17] in so much that a conscious representation of the object and it's component states are committed to memory. Given the limitations of working memory and that approximately 4 objects and their components states including colour information can simultaneously be recalled [18], [19]. In a complex environment with shifting focus, it is inevitable that much of the scene will not be recalled in detail. However, the perception of an object is related to the features of the object and its relationship to the contents of the VSTM and task goals [20]. Coherence theory [21] as a view of perception infers that in fact, the visual perception relies very little on actual memory and persistence after the withdrawing of attention as a scene can be sampled repeatedly and re-referenced at any given moment by the refocusing of attention [22]. Essentially the environment serves as "external memory". This external memory and its associated lack of processing is also referred to as distributed cognition [23], specifically where the cognitive work is offloaded to the environment. Essentially having the environment work to store and in some cases manipulate information, returning to collect only that data that later becomes pertinent.

Although many experiments have demonstrated that gradual change blindness can result in the non-detection of colour changes in otherwise static images, [24], [25] it has not been demonstrated what the results of changing broad environmental elements covering most of the visual field in a virtual environment would be. No known prior study has analysed the effect of colour, textural and object change across the visual field in a dynamic environment taking into account early and late changes to said properties [25]. No known prior study has analysed the impact that varying the duration of this change has either in such a dynamic environment and when duration has been investigated in static or smaller scenes [9] the time of change had been kept short under 
the assumption of an early attentive period. Inclusion of a range of durations allowed for the investigation of the potential role of later selection or distributed cognition [23] within dynamic, high cognitive load, environments and settings.

It was predicted that as gradual change is not consistently perceived therefore introducing such changes to all major materials in a virtual environment would not be noticed by the participants if distracted by a task requiring the utilisation of working memory.

The following hypotheses were considered:

1. There would be no significant effect of the time of change on the overall detection rate.

2. There would be no significant difference in the recollection of pre and post-state textural information.

\section{Method}

\section{$2.1 \quad$ Participants}

119 participants; 102 male, 17 female, age range 17 and $54(m=22.28$, SD = 6.36) all reporting having normal vision or corrected to normal vision. All participants had normal colour vision; those reporting a type of colour blindness were excluded from the test. Participants were recruited primarily from students and staff in the department of computing and engineering, they were not paid for their participation and did not receive course credits.

\subsection{Design}

A mixed design was employed with the time of the environmental changes (duration) acting as the between-subjects variable (11.25 seconds, 22.5 seconds, 45 seconds, 90 seconds). The details recalled at pre-change or post-change accurately served as the within-subjects variable. Participants were randomly assigned to one of the four duration conditions, and all participants were assessed on how many pre and post-change textural and object properties could be accurately recalled. This allowed for the impact of duration of change to be assessed, as well as any preferences for early (pre-change) or late (post-change) attention to environmental changes.

\subsection{Materials and Apparatus}

In order to test the hypotheses a virtual environment with a distraction task was created, this took the form of an 'escape the room' game created in Unreal Development Kit (UDK); consisting of a bedsit living space $12 \mathrm{~m}^{2}$ with en-suite bathroom $4 \mathrm{~m}^{2}$, a variety of objects such as computers, drawers and other objects could be interacted with and manipulated to solve a series of interrelated problems and eventually escape the room. 
Table 1. List of colour and textural changes made over time.

\begin{tabular}{lll}
\hline Object & Initial Texture & Post Change Texture \\
\hline Living-space walls & Cream with 1 dark stripe & Pink with 2 dark stripes \\
Flooring & Light Beech laminate & Dark Oak laminate \\
Sofa & Red & Brown \\
Bed/Duvet & Purple with dark throw & Pink with dark throw \\
Curtains & Red Un-patterned & Orange with flower print \\
Bathroom walls & Cream & Pink \\
Alice in Wonderland book & Blue with cat & Purple with just a smile \\
War of the Worlds book & Red & Green \\
The Time Machine book & Pink/Purple & Blue/Orange \\
A Princess of Mars book & Yellow & Orange \\
Heart of Darkness book & Red & Purple \\
Picture frame & Green & Blue \\
Picture of cliffs & A sea and cliff Scene & A Gorilla’s face is added \\
\hline
\end{tabular}

The puzzle aspect of the environment acted as the distraction from the simultaneous broad changes being made to the textures of most of the surfaces and objects in the room (see Table 1 and Fig. 1). Thirteen textures were altered ranging from major elements that filled most of the visual space to smaller objects such as books. The changes were not limited to hue shifts, but also included contrast changes and pattern changes. Example changes are shown in with specific examples from the living room and bedroom shown in Fig. 2 and Fig. 3 respectively. These textural changes were instigated at the moment the player entered the room and participants were engaged in the game for a total of 5 minutes. The changes described took place in the initial segment of that time and were static for the remaining time. 


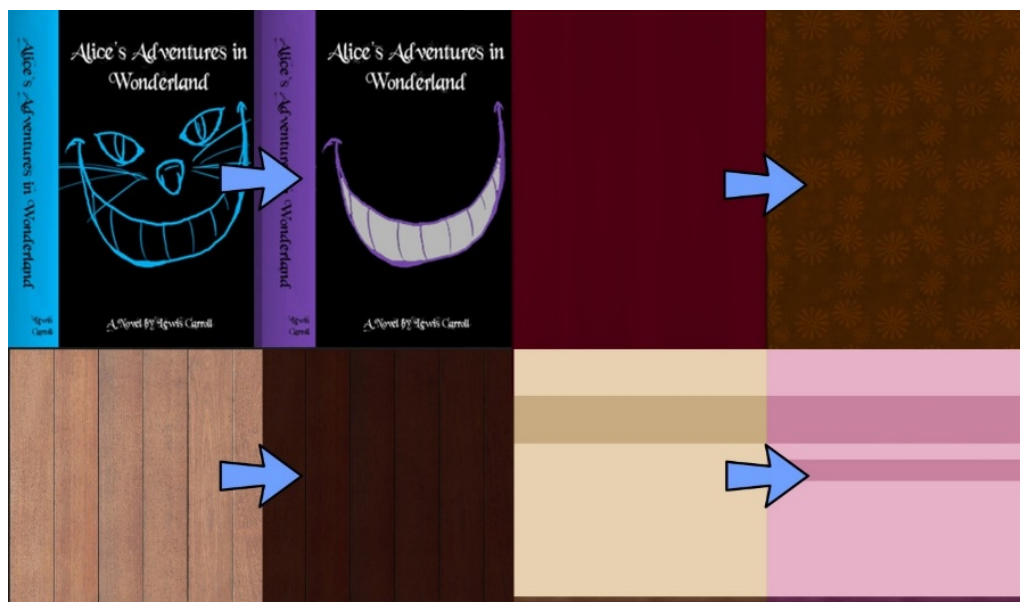

Fig. 1. Examples of variants of textural change, colour, contrast, pattern and image.

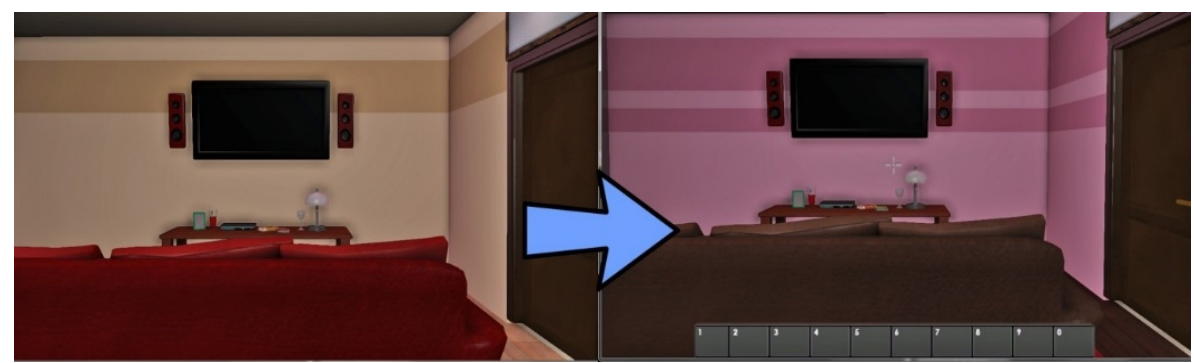

Fig. 2. The transition of textures in the living room area.

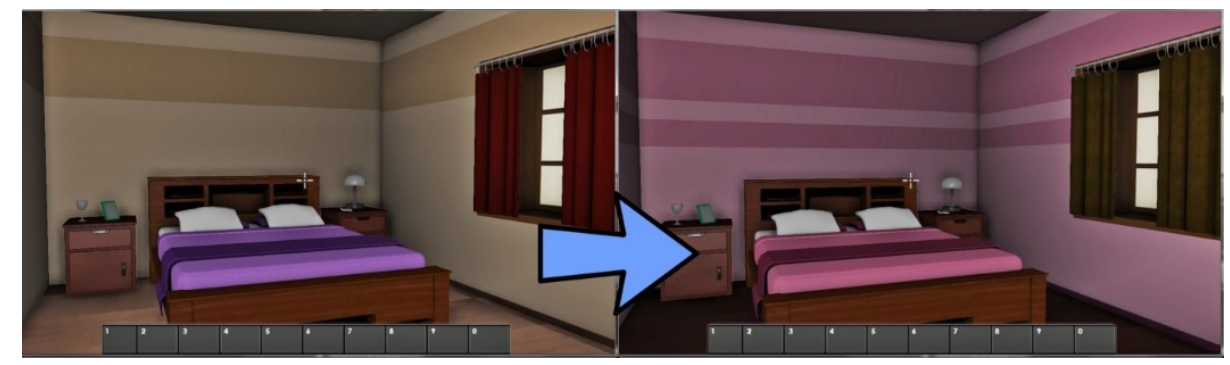

Fig. 3. The transition of textures in the bedroom area.

Following prior change blindness experiments [6], [26], an image of a gorilla was included in the scene; this was embedded into a prominent picture and gradually appeared at the same rate as the other changes. 


\subsection{Procedure}

The participants received instructions to solve the puzzle in the allocated time and no information about the nature of the test was provided to them. On-screen instructions explained basic interaction to the participants, after which they were immediately immersed in a virtual environment of a small single room apartment. The room contained several interactive objects to be examined, allowing the discovery of clues to progress the game. For instance, the computer on the desktop required a user name and password. Clues to this were contained on a post-it note on the desk and the password was the name of the artist in the painting. Further puzzles involved finding batteries to fit into a remote control unit in order that the TV could be switched on.

During their exploration of the environment, thirteen colour and/or pattern changes occurred to textures within the scene with the game remaining playable for a total of 5 minutes.

After 5 minutes of gameplay, the programme automatically ended the session and the participants were asked to immediately complete a post-test questionnaire. As participants were told that the experiment focused on gameplay, the questions began with queries relating to game design issues and slowly introduced specific questions about overall perception. The questions were designed so as not to lead the participant to provide a predetermined answer. The questions in order were;

- Briefly describe the main focus of the game

- What game genre does it belong to?

- Did you notice anything unusual or peculiar whilst playing?

- Did you notice anything change in the game?

- Please list any changes you think you noticed. Or simply type 'NONE' - following multiple-choice questions about the colours of particular objects, the final questions asked were:

— Did you spot the gorilla?

- If you answered YES to spotting the gorilla, where was it?

As the participants were naive to the aims of the experiment a general non-leading question about unusual events was asked first, followed by a more direct question regarding whether any change was noticed.

\section{Results}

$52.5 \%$ of participants claimed to have noticed a change, yet only $4.2 \%$ actually identified a manipulated gradual change, this being a total of 5 participants out of 120 (see Table 2). All other noticed changes were unrelated changes triggered by participant action (such as saying a door opened or items could be picked up and moved). Despite the questionnaire allowing for multiple entries in colour selection when asked to describe individual objects, none of the participants chose more than one colour option. 
Table 2. The changes detected in each of the time variants

\begin{tabular}{llll}
\hline $\begin{array}{l}\text { Duration of tex- } \\
\text { ture changes. }\end{array}$ & $\begin{array}{l}\text { Number of partici- } \\
\text { pants }\end{array}$ & $\begin{array}{l}\text { Number reporting } \\
\text { change detection }\end{array}$ & Percentage \\
\hline 90 seconds & 31 & 1 & $3.23 \%$ \\
45 seconds & 27 & 2 & $7.40 \%$ \\
22.5 seconds & 30 & 1 & $3.33 \%$ \\
11.25 seconds & 31 & 1 & $3.23 \%$ \\
Total & 119 & 5 & $4.20 \%$ \\
\hline
\end{tabular}

Table 3. Percentage identifications of gradual change item properties

\begin{tabular}{lllll}
\hline Change Item & $\begin{array}{l}\text { Identified Pre- } \\
\text { Change Col- } \\
\text { our }\end{array}$ & $\begin{array}{l}\text { Identified } \\
\text { Post-Change } \\
\text { Colour }\end{array}$ & $\begin{array}{l}\text { Incorrect } \\
\text { Identification }\end{array}$ & $\begin{array}{l}\text { Did Not } \\
\text { Know }\end{array}$ \\
\hline Curtains & 38.8 & 3.3 & 34.7 & 22.3 \\
$\begin{array}{l}\text { WotW Book } \\
\begin{array}{l}\text { Bedroom } \\
\text { Walls }\end{array}\end{array}$ & 9.1 & 15.7 & 21.5 & 53.3 \\
$\begin{array}{l}\text { Bathroom } \\
\text { Walls }\end{array}$ & 6.6 & 25.8 & 44.2 & 23.3 \\
$\begin{array}{l}\text { Bed Duvet } \\
\text { Mean Total }\end{array}$ & 20.8 & 6.6 & 61.7 & 25 \\
\hline
\end{tabular}

When asked if they had noticed a gorilla in the room $11.6 \%$ claimed to have done so, though only $9.9 \%$ were able to accurately state where they had seen the gorilla (in the painting). It is important to note this question only determined whether they had seen the gorilla at some point during their experience, not whether they noticed it had gradually appeared. When detailing the changes they had noticed, only 1 participant stated they had seen the gorilla appear.

Specific questions were asked about the items' gradual changes that were core to the virtual experience (items close to focal points and tasks/goals) or covered a large area of the visual field (walls). As can be seen from Table 3, and consistent with prior studies, a large proportion of participants could not identify the colour or texture property of the gradual change items. In terms of which colour or texture properties reported there was little difference between the pre-change property (16.38\%) and post-change property (15.94\%).

This can be further seen when considering the total number of pre-change $(\mathrm{M}=.8$, $\mathrm{SD}=.78)$ and post-change $(\mathrm{M}=.75, \mathrm{SD}=.83)$ properties recalled. 
When broken down by condition (11.25, 22.5, 45 and 90 seconds), the number of participants reporting something unusual related to the gradual change, correctly identifying a change, or accurately noticing the gorilla, there is no significant difference between groups.

Table 4. Total Percentage identifications of gradual change item properties by condition

\begin{tabular}{lll}
\hline Condition & $\begin{array}{l}\text { Identified Pre-Change } \\
\text { Properties }\end{array}$ & $\begin{array}{l}\text { Identified Post-Change } \\
\text { Properties }\end{array}$ \\
\hline 11.25 Seconds & 18.14 & 15.64 \\
22.5 Seconds & 12.01 & 17.34 \\
45 Seconds & 20.72 & 16.28 \\
90 Seconds & 15.48 & 14.86 \\
\hline
\end{tabular}

When considering the overall difference in pre and post-change identification across three of the four conditions the pre-change property is identified slightly more than the post-change, though the differences appear small, shown in Table 4. This is further supported by the mean number of pre and post-change properties identified as per Table 5.

Table 5. Mean total identification of pre and post-change properties by condition

\begin{tabular}{lll}
\hline Condition & Mean Pre-Change (SD) & Mean Post-Change (SD) \\
\hline 11.25 Seconds & $.87(.87)$ & $.68(1.02)$ \\
22.5 Seconds & $.56(.67)$ & $.86(.81)$ \\
45 Seconds & $1.03(.85)$ & $.77(.64)$ \\
90 Seconds & $.77(.64)$ & $.67(.79)$ \\
\hline
\end{tabular}

A mixed 4 X 2 ANOVA with duration serving as the between-subjects factor (11.25, 22.5, 45 and 90 seconds), and change property identification (pre-change, post-change) serving as the within-subjects factor showed no significant main effect condition $(\mathrm{F}(3$, $116)=.723, \mathrm{p}=.54)$, or change property identification $(\mathrm{F}(1,116)=.315, \mathrm{P}=.576)$. No significant interaction effect $(F(3,116)=1.31, p=.275)$ was found.

\section{Discussion}

The aims of this research were to investigate whether gradual environmental changes are perceived and whether the duration of any changes affect perception rates.

It has been previously demonstrated that either colour changes [9] or small changes to a broader scene result in significant non-detection of those changes [27]. However, the results of this research have suggested that when combined with a distraction task, even the broadest of changes; in this case fundamentally altering the textures of an entire environment were not detected. The results show that overall; fewer than $5 \%$ of participants perceived any of these changes and many of the larger changes made were 
not detected by any participants. Given the limitations of Visual Short-Term Memory [28], it is unsurprising that the participants provided incorrect responses when asked to identify specific colours of objects. In addition, only $9.9 \%$ of participants correctly identified the location of the gorilla (Illustrated in Fig. 4) in the scene when it was clearly in a prominent and incongruous position and in an item required to solve a portion of the puzzle indicates that, as previously reported [20], participants only commit to memory elements that they perceive to be beneficial to the goals.

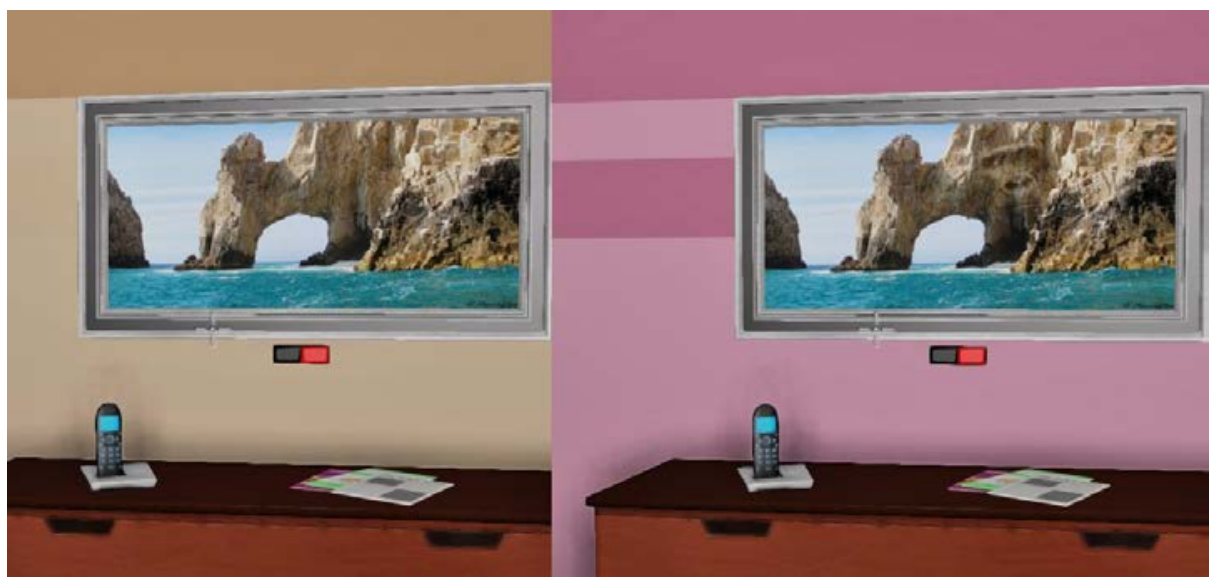

Fig. 4. Pre and post-change versions of the painting in which the face of a gorilla appears.

As previously demonstrated [9], small areas of static images can be changed over time and these changes are perceived approximately $30 \%$ of the time. It is clear that combined with a distraction task, and in a virtual representation of a scene, this detection of change when applied to the entire field of view falls away by approximately $90 \%$.

Memory for specific scene details correlates with current theories of memory; the results indicated that in many cases as the actual colour or texture of elements perceived to be background objects and not perceived as salient, no specific memory was encoded of the colour of those objects [20]. This explains the lack of significant difference in the amount of pre and post-change information accurately recalled by participants. This in turn further supports the notion that some of the cognitive load in these situations is distributed to the environment under the assumption that the environment is stable and consistent in its properties [23].

Within a video games context, the results of these experiments reflect a commonly utilised method of removing temporary visual information from a scene. In many instances, detail information is added to a scene in the form of bullet holes, blood splatter or footprints in the snow. These elements commonly disappear over time to reduce processing and memory overhead and unless the player is attending to them directly will disappear without the player noticing. 
While changes that include the removal of extraneous information will not be noticed and therefore will not break the flow of a game title. This research has demonstrated that broad changes can be gradually made to an environment without the player noticing these differences. From a design perspective, this understanding allows for the morphing of an entire environment. This feature may relate to narrative or define a specific sense of drama, mood, or mental state. It opens new areas for visual storytelling that have not yet been fully explored. Further research to could seek to identify if these results are replicated in virtual reality.

\section{References}

[1] T. N. Downs, "Modern Coin Manipulation,” pp. 1-105, 1900.

[2] S. Martinez-Conde and S. L. Macknik, "Magic and the brain,” Sci. Am., vol. 299, no. 6, pp. 72-79, 2008.

[3] D. Dennett, Consciousness Explained. Little, Brown and Co., 1991.

[4] U. Neisser and R. Becklen, "Selective looking: Attending to visually specified events," Cogn. Psychol., vol. 7, no. 4, pp. 480-494, 1975.

[5] R. Becklen and D. Cervone, "Selective looking and the noticing of unexpected events," Mem. Cognit., vol. 11, no. 6, pp. 601-608, 1983.

[6] D. J. Simons and C. F. Chabris, "Gorillas in our midst: sustained inattentional blindness for dynamic events.,” Perception, vol. 28, no. 9, pp. 1059-74, Jan. 1999.

[7] J. Grimes, "On the failure to detect changes in scenes across saccades.," Proc. R. Soc. London, pp. 89-110, 1996.

[8] S. J. Blackmore, G. Brelstaff, K. Nelson, and T. Trościanko, "Is the richness of our visual world an illusion? Transsaccadic memory for complex scenes.," Perception, vol. 24, no. 9, pp. 1075-1081, 1995.

[9] D. J. Simons, S. L. Franconeri, and R. L. Reimer, "Change blindness in the absence of a visual disruption,” Perception, vol. 29, no. 10, pp. 1143-1154, 2000.

[10] S. Garden, C. Cornoldi, and R. H. Logie, "Visuo-spatial working memory in navigation,” Appl. Cogn. Psychol., vol. 16, no. 1, pp. 35-50, Jan. 2002.

[11] T. C. W. Nijboer, R. Kanai, E. H. F. de Haan, and M. J. van der Smagt, "Recognising the forest, but not the trees: An effect of colour on scene perception and recognition," Conscious. Cogn., vol. 17, no. 3, pp. 741-752, 2008.

[12] M. Pilling and A. Gellatly, "Visual awareness of objects and their colour," Attention, Perception, Psychophys., vol. 73, no. 7, pp. 2026-2043, 2011.

[13] J. M. Wolfe, A. Reinecke, and P. Brawn, Why don't we see changes? The role of attentional bottlenecks and limited visual memory, vol. 14, no. 4-8. 2006.

[14] A. Baddeley, R. Logie, S. Bressi, S. Della Sala, and H. Spinnler, "Dementia and Working Memory,” Q. J. Exp. Psychol. Sect. A, vol. 38, no. 4, pp. 603-618, Nov. 1986.

[15] L. Pessoa and L. G. Ungerleider, "Neural Correlates of Change Detection and Change Blindness in a Working Memory Task,” Cereb. Cortex, vol. 14, no. 5, pp. 511-520, 2004.

[16] W. A. Phillips and A. D. Baddeley, "Reaction time and short-term visual memory*," 
Psychon. Sci., vol. 22, no. 2, pp. 73-74, 1971.

[17] V. A. F. Lamme, "Why visual attention and awareness are different.," Trends Cogn. Sci., vol. 7, no. 1, pp. 12-18, 2003.

[18] A. Hollingworth, C. C. Williams, and J. M. Henderson, “To see and remember: Visually specific information is retained in memory from previously attended objects in natural scenes,” Psychon. Bull. Rev., vol. 8, no. 4, pp. 761-768, 2001.

[19] S. J. Luck and E. K. Vogel, "The capacity of visual working memory for features and conjunctions,” Nature, vol. 390, no. 6657, pp. 279-284, 1997.

[20] A. Hollingworth, M. Matsukura, and S. J. Luck, "Visual Working Memory Modulates Rapid Eye Movements to Simple Onset Targets," Psychol. Sci., vol. 24, no. 5, pp. 790796, 2013.

[21] R. A. Rensink, “The dynamic representation of scenes,” Vis. cogn., vol. 7, no. 1-3, pp. 17-42, 2000.

[22] D. H. Ballard, M. M. Hayhoe, P. K. Pook, and R. P. N. Rao, "Deictic codes for the embodiment of cognition,” Behav. Brain Sci., vol. 20, no. 4, pp. 723-767, 1997.

[23] M. Wilson, “Six views of embodied cognition,” Psychon. Bull. Rev., vol. 9, no. 4, pp. 625-636, 2002.

[24] J. G. Arrington, D. T. Levin, and D. A. Varakin, "Color onsets and offsets, and luminance changes can cause change blindness,” Perception, vol. 35, no. 12, pp. 16651678, 2006.

[25] S. B. Most, D. J. Simons, B. J. Scholl, and C. F. Chabris, "Sustained Inattentional Blindness : Dynamic Events,” Psyche (Stuttg)., vol. 6, no. 14, pp. 1059-1074, 2000.

[26] T. Drew, M. L. H. Võ, and J. M. Wolfe, "The Invisible Gorilla Strikes Again: Sustained Inattentional Blindness in Expert Observers,” Psychol. Sci., vol. 24, no. 9, pp. 18481853, Sep. 2013.

[27] S. L. Franconeri, A. Hollingworth, and D. J. Simons, "Do new objects capture attention?,” Psychol. Sci., vol. 16, no. 4, pp. 275-281, 2005.

[28] A. D. Baddeley and G. Hitch, "Working Memory,” Psychol. Learn. Motiv., vol. 8, pp. 47-89, 1974. 